\title{
A Novel $\mu$-Negative Metamaterial With Enhanced Rejection Bandwidth
}

\author{
${ }^{1}$ Haider Raad, ${ }^{2}$ Hussain Al-Rizzo, ${ }^{2}$ Ayman Abbosh and ${ }^{2}$ Said Abushamleh \\ ${ }^{1}$ Department of Engineering Science, College of Science and Technology, \\ Sonoma State University, Rohnert Park, California, USA \\ ${ }^{2}$ Department of Systems Engineering, College of Engineering and Technology, \\ University of Arkansas at Little Rock, Little Rock, Arkansas, USA
}

Received 2013-01-04, Revised 2013-04-13; Accepted 2013-05-03

\begin{abstract}
In this study, a novel planar $\mu$-Negative (MNG) metamaterial structure based on Split Ring Resonators (SRRs) with improved rejection bandwidth is presented. A bridging technique is used to connect two SRR unit cells at the center to emulate a cascaded filter. The proposed structure achieved a $-20 \mathrm{~dB}$ rejection bandwidth of $15.5 \%$ compared to $4.5 \%$ for the conventional SRRs which makes it a good candidate for integration with antennas and RF circuits that require large operational bandwidth.
\end{abstract}

Keywords: $\mu$-Negative Metamaterial, Split Ring Resonator, RF Circuits, Wireless Systems

\section{INTRODUCTION}

The past decade has witnessed the revolution of Metamaterials (MTMs), artificially engineered structures which exhibit unique electromagnetic properties that do not exist in nature. These materials can be engineered to have desired features within a specified frequency range. The integration of these artificial structures with antennas and microwave circuits has shown to be extremely beneficial in several applications (Pyo et al., 2010). Amongst these artificial materials, MNG metamaterials are of special interest due to the advantageous applications in the fields of antennas and $\mathrm{RF}$ systems. One of the most interesting features of MNGs is the possibility of synthesizing a magnetic material with a negative effective permeability at a specific frequency band in the microwave region. This is in contrast to conventional magnetic materials (i.e., ferrites) which are only magnetically operational in the UHF region and below. Obviously, modern wireless systems such as: L-band, Industrial Scientific Medical (ISM) and UWB bands would highly benefit from such MNG materials. The idea of synthesizing artificial magnetic materials from conductors was first proposed by Schelkunoff and Friis (1952). Ziolkowski and Auzanneau (1997) proposed the idea of a magnetic wall based on artificial molecules, while Sievenpiper invented the AMC which operates as a surface rather than a medium in 1999. Eventually, Pendry et al. (1999) came up with a MNG material based on SRR structures which have become the cornerstone of MTMs and one of the most widely applied artificial magnetic materials today.

Pendry's SRR comprises two concentric metallic rings printed on a substrate, with splits in the rings etched out at opposite sides. Later on, a plethora of artificial magnetic structures have been proposed in the literature. Marques et al. (2002) proposed a modified version of Pendry's SRR (hence called Modified SRR (MSRR)), where the two rings exhibit increased capacitance through broadside capacitive coupling which is higher than the edge coupling in the latter. Although the SRR structures are electrically small (i.e., largest unit cell dimension $<<\lambda$ ), further miniaturization is required for compact and low profile applications. A number of miniaturized artificial magnetic inclusions have been proposed in the literature, spiral resonators, capacitivelySonoma State University, Rohnert Park, California, USA 
loaded loops and space-filling (Hilbert) curves, to mention a few (Erentok et al., 2005; McVay et al., 2004; Yousefi and Ramahi, 2010).

One of the main advantages of using magnetic materials $(\mu>1)$ at microwave frequencies is to enhance the impedance bandwidth and allow miniaturization of radiating elements since the effective length of the radiating element scales down with $1 \sqrt{\mu}$. Bilotti et al. (2006), proposed MNG structures embedded in a circular patch antenna's substrate to excite sub-wavelength radiating modes which are independent of the patch's size. Miniaturization of microstrip antennas with substrate loading by spiral resonators is investigated by (Baena et al., 2004). The dimensions of the proposed design are substantially reduced at the cost of antenna's efficiency. Another interesting application of MNGs, is the mitigation of surface wave propagation between radiating elements (since EM wave propagation is prohibited in media with a negative value of either $\mu$ or ع. Bait-Suwailam et al. (2010) utilized six sets of SRR arrays to reduce mutual coupling between two high profile monopoles. As is well known, MNG media can be realized through SRR structures. When the SRRs are excited by an incident electromagnetic wave with a magnetic field parallel to their axes, currents will be induced on both the inner and outer rings and charges accumulate across the gaps. Consequently, the SRR structures act as magnetic dipoles and an effective MNG medium is obtained which prevents propagating modes within a specific frequency band. This behavior can be represented by a matrix of LC circuits with inductance $\mathrm{L}$ (represented by the current paths in the metallic rings) and capacitance $C$ (represented by the gaps between the rings). Hence, SRR structures can be characterized by the effective capacitance and inductance with a resonant frequency $\omega_{\mathrm{m}}=1 \sqrt{\mathrm{LC}}$ and a negative permeability can be achieved within a narrow frequency band slightly above the resonant frequency $\omega_{\mathrm{m}}$ as will be explained.

Complementary Split Ring Resonator (CSRR) structures are considered as the dual counterpart of SRR since they exhibit a resonant behavior when subjected to a vertically polarized electric field.

A slotted CSRR structure is proposed by Swailam et al. (2010) to effectively reduce the mutual coupling between two square microstrip antennas. The slot combining two unit cells is proven to enhance the bandwidth and the stop band behavior. By applying the duality principle, a dual structure is proposed in this study where SRR unit cells are bridged to enhance the stop band performance when magnetic fields are dominantly vertically polarized. Such combined structures can be thought of as cascaded filters (Swailam et al., 2010).

A theoretical background on $\mu$-Negative Metamaterial is provided. We discuss the design and methodology of the proposed structure. Results will be discussed and finally, conclusions are given.

\section{1. $\mu$-Negative Metamaterial: Theoretical Background}

MNG metamaterial structures are sensitive to the orientation of the impinging magnetic field vector. An electromotive force (emf) is excited by a magnetic field parallel to the SRRs axis. Consequently, an induced current circulates along each ring of the SRRs. In addition, the capacitive gaps between the rings allow displacement currents exchange between the rings. The total current induces an opposite magnetic field. The total induced current I can be expressed as (Ikonen et al., 2004) Equation 1:

$$
I=\frac{e m f}{z_{\text {SRR }}} \frac{e m f}{j \omega L_{a} f f+\frac{1}{j \omega C_{a} f f}+R}
$$

where, $Z_{\mathrm{SRR}}$ is the total impedance of the SRR. The structure circuit parameters $\mathrm{L}_{\text {eff }}, \mathrm{C}_{\text {eff }}, \mathrm{R}$ represent the inductance expressed by the current path in the rings, capacitance (induced in the gaps and between the inner and outer rings) and the resistance of the structure represented by the finite conductivity of the metallic material (copper, silver). The electromotive force can be expressed as Equation 2:

$\mathrm{emf}=-\mathrm{j} \omega \mu_{\mathrm{eff}} \mathrm{SH}_{\mathrm{ext}}$

where, $\mathrm{S}$ is the effective area impinged by the external magnetic field, $\mathrm{H}_{\text {ext }}$.

The induced magnetic field will exert a magnetic dipole moment within the structure Equation 3:

$\mathrm{m}=\mu_{\text {eff }} \mathrm{SI}$

where, $\mathrm{m}$ is the magnetic dipole moment of an individual SRR structure. The magnetic susceptibility, $X_{m}$, is a measure of the magnetization in a material due to an applied magnetic field. It can be expressed as Equation 4:

$\mathrm{x}_{\mathrm{m}}=\frac{\mathrm{M}}{\mathrm{H}_{\mathrm{axt}}}$

where, $\mathrm{M}$ is the average moment of the magnetic dipoles within the bulk homogeneous structure. 


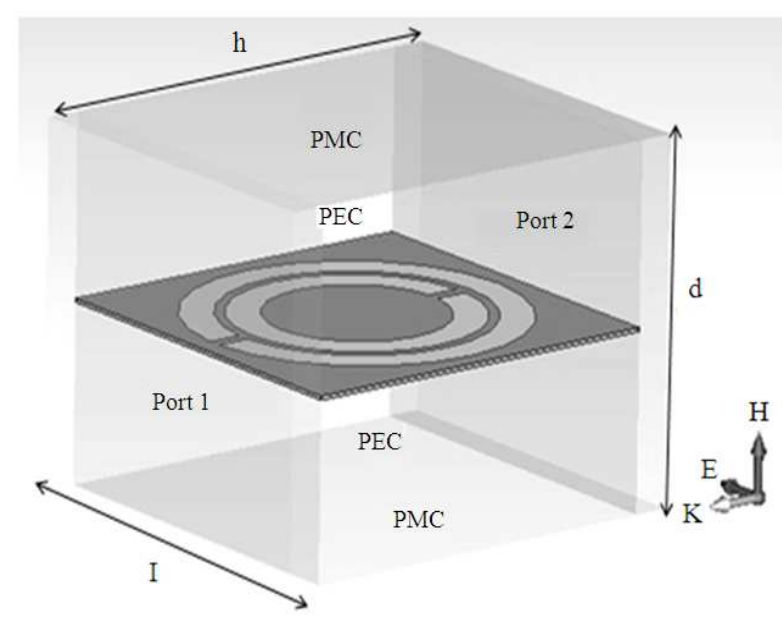

Fig. 1. A SRR structure positioned at the center of a pa-rallelplate waveguide (Khaleel et al., 2012)

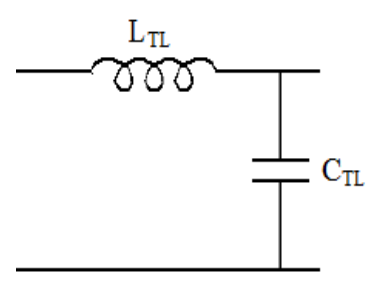

(a)

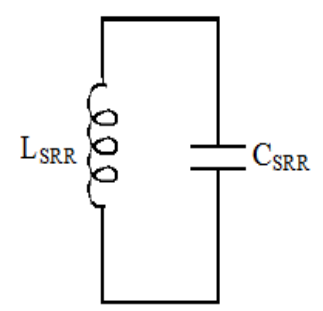

(b)
Fig. 2. A circuit model for (a) parallel-plate waveguide (b) SRR structure

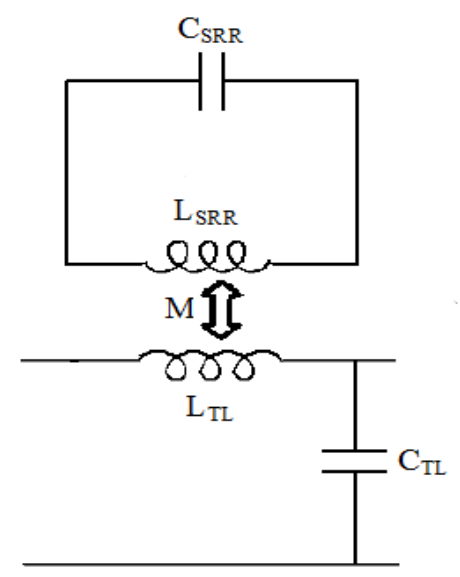

Fig. 3. A circuit model of the SRR in the parallel-plate waveguide

The permeability of a magnetic material is generally expressed as the ratio of the total magnetic flux density and the applied magnetic field Equation 5: $\mu_{\text {eff }}=\mu_{\text {eff }}\left(1+X_{\mathrm{m}}\right)$

From the above equation, it is evident that the effective permeability can be enhanced by increasing the effective magnetic susceptibility. It should be noted that the effective permeability is complex, with a positive real part slightly below the structure's resonant frequency and a negative real part slightly above resonance.

A more in-depth analysis of such structures can be obtained from a macroscopic circuit model. An accurate circuit model reported by (Grbic, 2005; Martin et al., 2003 ) is used to analyze and demonstrate the resonant nature of SRR structure. As depicted in Fig. 1, the structure is excited by an axial magnetic field positioned at the center of a fictitious parallel-plate waveguide which allows a unit cell to be duplicated infinitely emulating a periodic structure.

A well known circuit model for a parallel-plate waveguide which comprises a series inductance and a shunt capacitance is depicted in Fig. 2a. The series inductance is represented by Equation 6:

$\mathrm{L}_{\mathrm{TL}}=\frac{\mu_{\mathrm{eff}} \mathrm{A}}{\mathrm{d}}$

where, $\mathrm{A}=\mathrm{lh}$, while the shunt capacitance is given by Equation 7:

$\mathrm{C}_{\mathrm{TL}}=\frac{\mu_{\mathrm{eff}} \mathrm{id}}{\mathrm{h}}$

As mentioned previously, the SRR possesses an inductance from the two rings paths and a capacitance from the gap which can be modeled as an LC resonator as shown in Fig. 2b. An induced current on the SRR is formed by the magnetic field of the Transverse Electromagnetic (TEM) mode in the parallel-plate waveguide which in turn sets up a voltage across the capacitive gap. This voltage can be simply comprehended by applying Faraday's law Equation 8:

$\mathrm{V}_{\mathrm{SRR}}=\frac{-\mathrm{d} \phi \mathrm{B}}{\mathrm{dt}}$

where, $V_{\text {SRR }}$ is the voltage across the SRR's capacitive gap that is induced by the axial magnetic field, $\mathrm{H}$ and $\phi_{\mathrm{B}}$ is the magnetic flux within the SRR. This can be represented in the phasor form as Equation 9:

$\mathrm{V}_{\mathrm{SRR}}=\mathrm{j} \omega \mathrm{A}_{\mathrm{SRR}} \mu_{\mathrm{eff}} \mathrm{H}$ 
where, $\phi_{\mathrm{B}}$ is replaced by $\mathrm{A}_{\mathrm{SRR}} \mu_{\mathrm{eff}} \mathrm{H}$ where $\mathrm{A}_{\mathrm{SRR}}$ is the loop area of the SRR structure. The interaction between the axial magnetic field enforced in the parallel-plate waveguide and the SRR loops can be represented by a mutual inductance $M$ in a circuit model as shown in Fig. 3. The induced voltage across $\mathrm{L}_{\mathrm{SRR}}$ by the mutual inductance can be expressed by $\mathrm{j} \omega \mathrm{MI}_{1}$, where $\mathrm{I}_{1}$ is the current flowing through $\mathrm{L}_{\mathrm{TL}}$. By equating this expression to the right side of (5) and applying Ampere's law on the current flowing through $\mathrm{L}_{\mathrm{TL}}\left(\mathrm{I}_{1}=\mathrm{Hd}\right)$, then $\mathrm{M}$ can be represented as follows Equation 10:

$\mathrm{M}=\frac{\mu \operatorname{effA}_{\mathrm{SRR}}}{\mathrm{d}}$

Applying circuit analysis to the model depicted in Fig. 3, the following set of equations can be derived Equation 11-13:

$\mathrm{V}_{1}=\mathrm{j} \omega \mathrm{L}_{\mathrm{TL}} \mathrm{I}_{1}+\mathrm{j} \omega \mathrm{MI}_{2}$

$\mathrm{V}_{2}=\mathrm{j} \omega \mathrm{MI}_{1}+\mathrm{j} \omega \mathrm{L}_{\mathrm{SRR}} \mathrm{I}_{2}$

$\mathrm{V}_{2}=\frac{\mathrm{j}}{\omega \mathrm{C}_{\mathrm{SRR}}} \mathrm{I}_{2}$

where, $\mathrm{V}_{1}$ is the voltage across $\mathrm{L}_{\mathrm{TL}}, \mathrm{V}_{2}$ is the voltage across $L_{S R R}$ and $I_{2}$ is the current on the loops path of the SRR. By further simplifications to the above equations, the impedance of the series branch $Z_{\text {series }}=V_{1} / I_{1}$ can be obtained Equation 14:

$$
Z_{\text {series }}=j \omega L_{T ~}-\frac{j \omega^{2} M^{2}}{\omega L_{S R R}-\frac{1}{\omega C_{\text {SRR }}}}
$$

To extract the effective permeability, $\mu_{\text {eff }}$ of the SRR, it can be shown that the series impedance of a parallel-plate waveguide filled with the SRR structures is represented by Equation 15:

$Z_{\mathrm{ppw}}=\frac{j \omega \mu \mathrm{eff} A}{\mathrm{~d}}=\frac{j \omega \mathrm{L}_{\mathrm{TL}} \mu \mathrm{eff}}{\mu \mathrm{eff}}$

Thus by equating (13) with (14), the effective permeability can be given by Equation 16-18:

$$
\mathrm{Z}_{\mathrm{ppw}}=\mathrm{Z}_{\text {series }}
$$

$$
\begin{aligned}
& \frac{\mu \text { eff }}{\mu \text { eff }}=\frac{\text { zseries }}{j \omega \mathrm{L}_{\mathrm{TL}}} \\
& \frac{\mu \mathrm{eff}}{\mu \mathrm{eff}}=1-\frac{\omega \mathrm{M}^{2}}{\mathrm{~L}_{\mathrm{TL}}\left(\omega \mathrm{L}_{\mathrm{SRR}}-\frac{1}{\omega \mathrm{C}_{\mathrm{SRR}}}\right.}
\end{aligned}
$$

The above expression represents the effective permeability of a periodic array of SRR structures, which follows a Lorentzian type dispersion (Smith et al., 2002). The inclusion's resonant frequency can be extracted using Equation 19:

$\omega_{\mathrm{mp}}=\frac{1}{\sqrt{\mathrm{L}_{\mathrm{SRR}} \mathrm{C}_{\mathrm{SRR}}}}$

where, the SRR is of a capacitive nature below $\omega_{\mathrm{mp}}$ and inductive above. Due to the capacitive nature of the SRR, as $\omega \rightarrow \omega_{\mathrm{mp}}$, the effective permeability increases positively, while at resonance, $\omega=\omega_{\mathrm{mp}}, \mu_{\mathrm{eff}}$ switches signs and turns negative. As $\omega$ gets farther away from $\omega_{\mathrm{mp}}, \mu_{\text {eff }}$ becomes less negative until $\omega$ approaches $\omega_{\mathrm{mp}}$, where $\omega_{\mathrm{mp}}$ is the plasma frequency of the SRR expressed by Equation 20:

$$
\omega_{\mathrm{mp}}=\frac{\omega_{\mathrm{mp}}}{\sqrt{1-\frac{\mathrm{M}^{2}}{\mathrm{~L}_{\mathrm{SRR}} \mathrm{L}_{\mathrm{TL}}}}}
$$

At this frequency $\mu_{\text {eff }}=0$. This demonstrates that the reactive loading of the SRR by the magnetic field generates an effective permeability which becomes negative and switches signs at a frequency band constrained by design. It is worth mentioning that resonant modes exist other than the one expressed above. These resonances take place due to higher order, nonuniform current distributions sustained by the SRR structure, either by one of the rings or by the structure as a whole. These higher order resonances can also give rise to an effective permeability and permittivity due to electrical coupling and can be utilized separately.

\section{MATERIALS AND METHODS}

The dimensions are chosen such that the structure resonates at the frequency range under consideration $(2.45 \mathrm{GHz})$. Figure 4a depicts a unit cell of the proposed BSRR which is modeled to have an effective negative permeability around $2.45 \mathrm{GHz}$. 


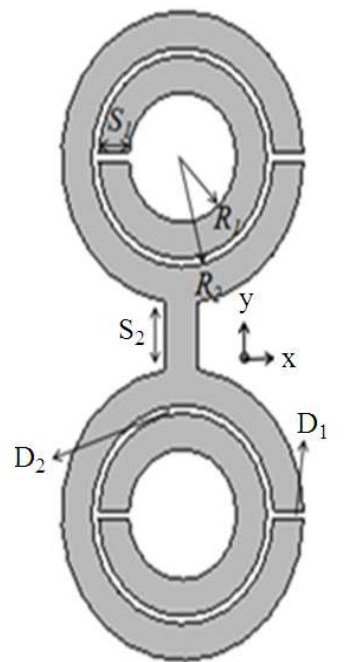

(a)

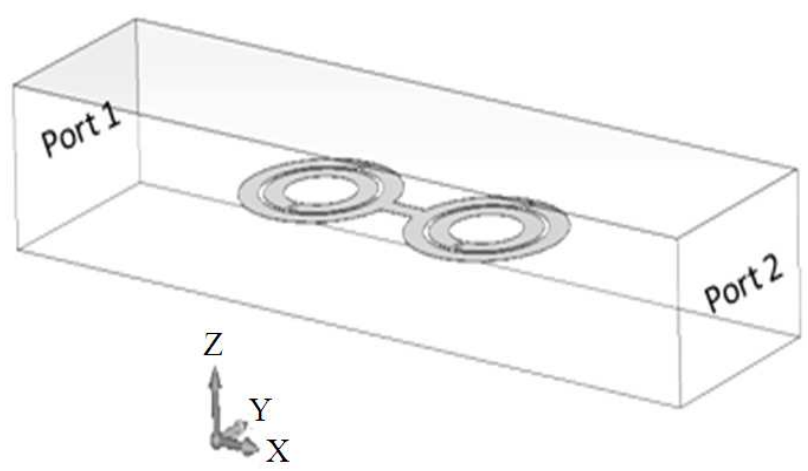

(b)

Fig. 4. Unit cell geometry of the proposed flexible BSRR (a) and numerical setup based on the virtual waveguide method used for the characterization of MNG metamaterial (b). PMC boundary conditions are assigned to the top and bottom of the waveguide while

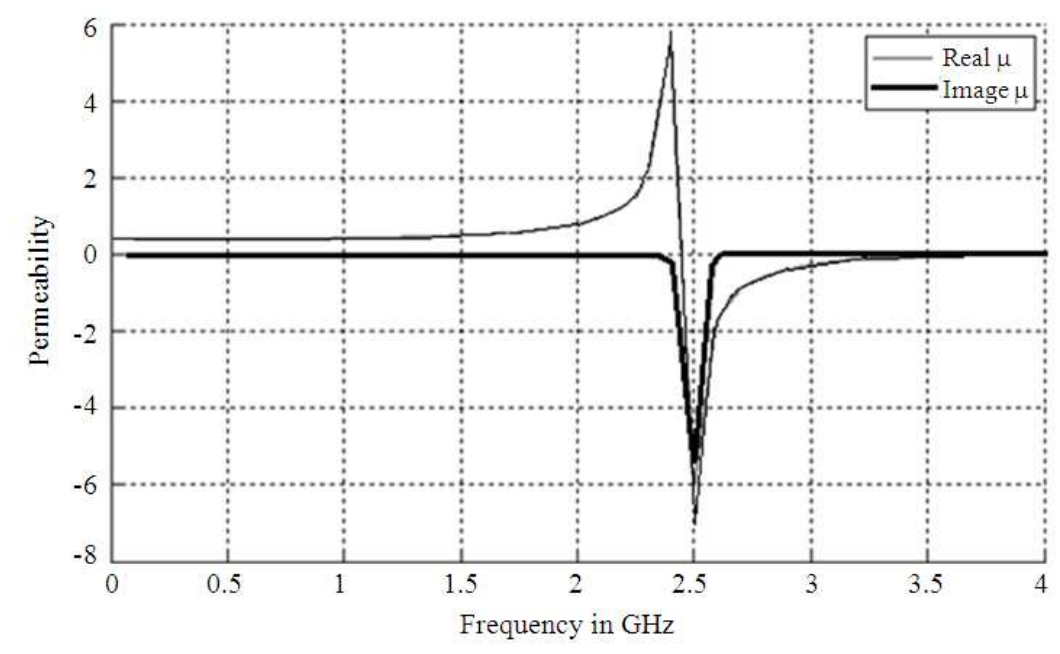

Fig. 5. Effective complex permeability values the pro-posed BSRR unit cell

The dimensions of the BSRR to operate at the desired resonant frequency are as follows: $\mathrm{R} 1=3 \mathrm{~mm}$, $\mathrm{R} 2=4.9 \mathrm{~mm}, \mathrm{~S} 1=1.63 \mathrm{~mm}, \mathrm{~S} 2=3 \mathrm{~mm}, \mathrm{D} 1=0.4 \mathrm{~mm}$ and $\mathrm{D} 2=0.3 \mathrm{~mm}$. In order to characterize the SRR model, the scattering parameters of the unit cell must be obtained. A BSRR unit cell is positioned at the center of a waveguide excited by an incident wave with $\mathrm{E}$ and $\mathrm{H}$ fields oriented as described in Fig. 4b where Perfect Electric Conductor (PEC) boundary conditions are assigned to the side walls of the waveguide, while
Perfect Magnetic Conductor (PMC) boundaries are assigned to the top and bottom walls in order to enforce the TEM. Generally, the effective permeability and permittivity are calculated through the refractive index and impedance matrix which are extracted from the scattering parameters of a SRR unit cell. A general procedure to retrieve the complex constitutive parameters of metamaterial structures is reported by (Chen et al., 2004). First, the S-parameters (both reflection and transmission coefficients) are extracted 
from a full wave simulation as depicted in Fig. 4. Next, the refractive index, $\mathrm{n}$ and the impedance, $\mathrm{Z}$, are obtained from the S-parameter inversion given by Equation 21-23:

zeff $=1) \pm \sqrt{\frac{\left(1+S_{11}\right)^{2}}{\left(1-S_{11}\right)^{2}}}$

$\mathrm{e}(-\mathrm{j} \eta \mathrm{effkd})=\mathrm{X} \pm \sqrt{1-\mathrm{X}^{2}}$

Where:

$$
\mathrm{X}=\frac{1}{2 \mathrm{~s}_{21}\left(1-\mathrm{s}_{11}^{2}+\mathrm{s}_{21}^{2}\right)}
$$

where, $\mathrm{d}$ is the largest dimension in the unit cell. It should be noted that since the metamaterial structure considered in this study is a passive medium, the signs in (21) and (22) are chosen such that Real $\left(Z_{\text {eff }}\right)>0$ and Im $\left(\eta_{\text {eff }}\right)<0$. Finally, the effective constitutive parameters $\varepsilon_{\text {eff }}$ and $\mu_{\text {eff }}$ are obtained by Equation 24 and 25:

$\mu_{\text {eff }}=\eta_{\text {eff }} Z_{\text {eff }}$

$\varepsilon_{\text {eff }}=\frac{\eta_{\text {eff }}}{z_{\text {eff }}}$
Figure 5 depicts the retrieved complex permeability of the BSRR structure.

\section{RESULTS AND DISCUSSION}

To demonstrate the effectiveness of the Bridged SRR (BSRR) in comparison to the conventional SRR structure, a numerical comparative study is conducted where the scattering parameters of a microstrip transmission line positioned over a BSRR unit cell, with and without the joining bridge are obtained. It is evident from Fig. 6 that the proposed structure exhibits an enhanced stop band performance over the conventional SRR. Figure 7 depicts the numerical setup for the microstrip transmission line method.

To explain the wide band behavior of the proposed structure, a duality principle can be applied to the slotted Complimentary Split Ring Resonator (CSRR) structure which was proven to effectively increase the operational bandwidth compared to its single structure counterpart. Such combined structures can be thought of as cascaded filters. By applying the duality principle, the proposed structure can achieve similar bandwidth characteristics given that it is excited by a magnetic field component parallel to its axis (when magnetic fields are dominantly vertically polarized) which is the opposite case for the CSRR structure that would be only excited by an electric field vector parallel to its axis.

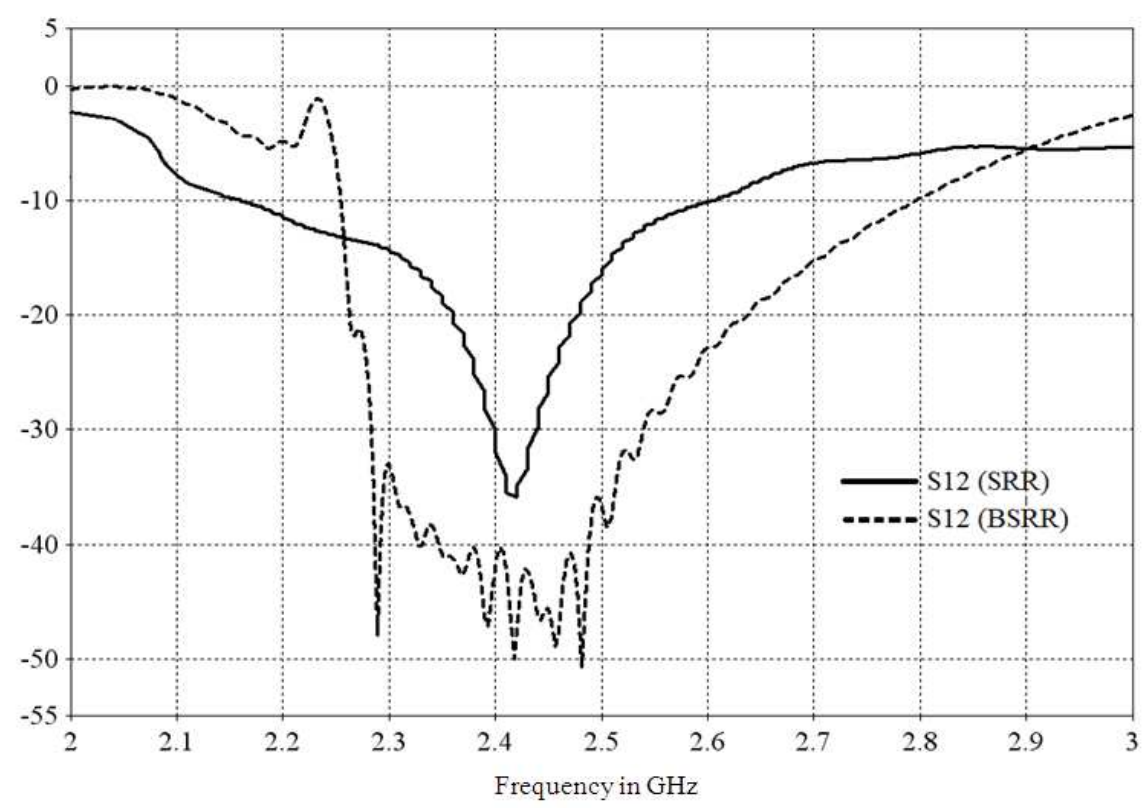

Fig. 6. Transmission coefficient S12 for the BSRR structure compared to a conventional SRR obtained from the transmission line method 


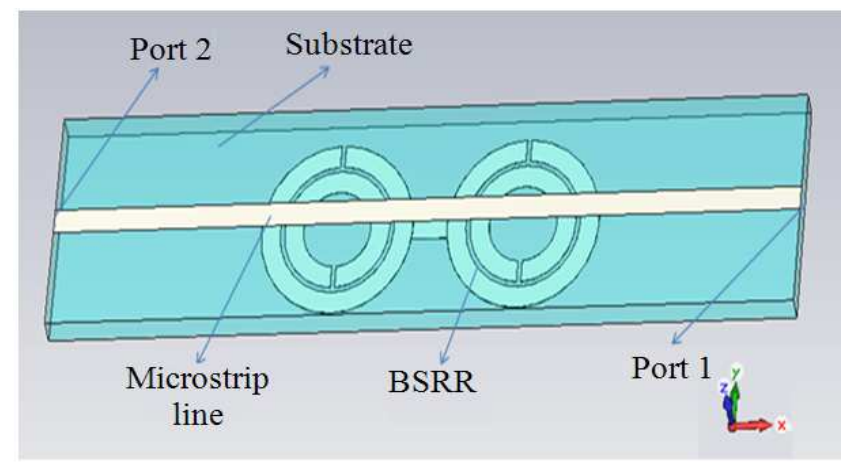

Fig. 7. Numerical setup for the microstrip transmission line method

\section{CONCLUSION}

In this study, a novel planar MNG structure composed of two SRRs connected at the center is presented. The bridging technique is proposed to enhance the rejection bandwidth by emulating a cascaded filter. The proposed structure achieved a -20 $\mathrm{dB}$ rejection bandwidth of $15.5 \%$ compared to $4.5 \%$ for the conventional SRRs which reflects a $32 \%$ increase in the stop bandwidth. This finding suggests that the structure would be a good candidate for integration with large-bandwidth RF circuits and antenna elements. Furthermore, a detailed theoretical analysis of $\mathrm{MNG}$ metamaterial was provided.

\section{REFERENCES}

Baena, J.D., R. Marques, F. Medina and J. Martel, 2004. Artificial magnetic metamaterial design by using spiral resonators. Phys. Rev. B, 69: 014402-014402. DOI: 10.1103/PhysRevB.69.014402

Bait-Suwailam, M.M., M.S. Boybay and O.M. Ramahi, 2010. Electromagnetic coupling reduction in highprofile monopole antennas using single-negative magnetic metamaterials for MIMO applications. IEEE Trans. Antennas Propagation, 58: 2894-2902. DOI: 10.1109/TAP.2010.2052560

Bilotti, F., A. Alu, N. Engheta and L. Vegni, 2006. Miniaturized circular patch antenna with metamaterial loading. Proceedings of the $1 \mathrm{st}$ European Conference on Antennas and Propagation, Nov. 6-10, IEEE Xplore Press, Nice, pp: 1-4. DOI: 10.1109/EUCAP.2006.4584634

Chen, X., T.M. Grzegorczyk, B. Wu, J. Pacheco and J.A. Kong, 2004. Robust method to retrieve the constitutive effective parameters of metamaterials. Phys. Rev. E, 70: 016608-016608. DOI: 10.1103/PhysRevE.70.01660
Erentok, A., P.L. Luljak and R.W. Ziolkowski, 2005. Characterization of a volumetric metamaterial realization of an arti cial magnetic conductor for antenna applications. IEEE Trans. Antennas Propag., 53: 160-172. DOI: 10.1109/TAP.2004.840534

Grbic, A., 2005. Super-resolving negative-refractiveindex transmission-line lenses. Ph.D. Theses, University of Toronto.

Ikonen, P., S. I. Maslovski, S. A. Tretyakov and I. A. Kolmakov, 2004. C material for microwave applications. Proceedings of the Progress in Electromagnetics Research Symposium, (PERS' 04), Pisa, Italy, pp: 485-488.

Khaleel, H.R., H. Al-Rizzo and D. Rucker, 2012. Effects of bending on the performance of split ring resonators. Microwave Opt. Technol. Lett., 54: 2098-2101. DOI: 10.1002/mop.27011

Marques, R., F. Medina and R. Rai-El-Idrissi, 2002. Role of bianisotropy in negative permeability and lefthanded metamaterials. Phys. Rev. B, 65: 144-146. DOI: 10.1103/PhysRevB.65.144440

Martin, F., J. Bonache, F. Falcone, M. Sorolla and R. Marques, 2003. Split ring resonator-based lefthanded coplanar waveguide. Applied Phys. Lett., 83: 4652-4654. DOI: 10.1063/1.1631392

McVay, J., N. Engheta and A. Hoorfar, 2004. High impedance metamaterial surfaces using Hilbertcurve inclusions. IEEE Microwave Wireless Comput. Lett., 14: 130-132. DOI: 10.1109/LMWC.2003.822571

Pendry, J.B., A.J. Holden, D.J. Robbins and W.J. Stewart, 1999. Magnetism from conductors and enhanced nonlinear phenomena. IEEE Trans. Microwave Theory Techniques, 47: 2075-2084. DOI: $10.1109 / 22.798002$ 
Pyo, S., S.M. Han, J.W. Baik, W.S. Yoon and Y.S. Kim, 2010. Offset-fed metamaterial antenna for radiation mode generation with efficiency improvement. Microwaves, Antennas Propagation, IET, 4: 1481-1490. DOI: 10.1049/iet map.2009.0245

Schelkunoff, S. and H.T. Friis, 1952. Antennas: Theory and Practice. 1st Edn., John Wiley and Sons, New York, pp: 639.

Smith, D.R., S. Schultz, P. Markos and C. M. Soukoulis, 2002. Determination of effective permittivity and permeability of metamaterials from reflection and transmission coefficients. Phys. Rev. B, 65: 95-104. DOI: 10.1103/PhysRevB.65.195104
Swailam, S., Z. Abdel-Salam, S. Emil, H. Nabil and W. Nammas, 2010. Multi-slice computed tomography: Can it adequately rule out left main coronary disease in patients with an intermediate probability of coronary artery disease? Cardiol. J., 17: 594-598.

Yousefi, L. and O.M. Ramahi, 2010. Arti_cial magnetic materials using fractal Hilbert curves. IEEE Trans. Antennas Propag., 58: 2614-2622. DOI: 10.1109/TAP.2010.2050438

Ziolkowski, R.W. and F. Auzanneau, 1997. Artificial molecule realization of a magnetic wall. J. Applied Phys., 82: 3192-3194. DOI: 10.1063/1.365624 